\title{
Construct Weak Hopf Algebras By Using Borcherds Matrix
}

\author{
Wu Zhixiang* \\ Mathematics Department, \\ Zhejiang University,Hangzhou, 310027, P.R.China
}

\begin{abstract}
We define a new kind quantized enveloping algebra of a generalized Kac-Moody algebra $\mathcal{G}$ by adding a new generator $J$ satisfying $J^{m}=J$ for some integer $m$. We denote this algebra by $w U_{q}^{\tau}(\mathcal{G})$. This algebra is a weak Hopf algebra if and only if $m=2,3$. In general, it is a bialgebra, and contains a Hopf subalgebra. This Hopf subalgebra is isomorphic to the usually quantum envelope algebra $U_{q}(\mathcal{G})$ of a generalized Kac-Moody algebra $\mathcal{G}$.
\end{abstract}

Keywords: generalized Kac-Moody algebras, irreducible representation, weak Hopf algebra

\section{INTRODUCTION}

In his study of Monstrous moonshine ${ }^{[3-5]}$, Borcherds introduced a new class of infinite dimensional Lie algebras called generalized Kac-Moody algebras. These generalized Kac-Moody algebra have a contravariant bilinear form which is almost positive definite. The fixed point algebra of any Kac-Moody algebra under a diagram automorphism is a generalized Kac-Moody algebra. A generalized Kac-Moody algebra can be regarded as a Kac-Moody algebra with imaginary simple roots. More explicitly, a generated Kac-Moody algebras is determined by a Borcherds-Cartan matrix $A=\left(a_{i j}\right)_{(i, j) \in I \times I}$, where either $a_{i i}=2$, or $a_{i i} \leq 0$. If $a_{i i} \leq 0$, then the index $i$ is called imaginary, and the corresponding simple root $\alpha_{i}$ is called imaginary root. In this paper, the set $\left\{i \in I \mid a_{i i}=2\right\}$ is denoted by $I^{+}$. Set $I^{i m}=I \backslash I^{+}$. The structure and the representation theory of generalized Kac-Moody algebras are very similar to those of Kac-Moody algebras, and many basic facts about Kac-Moody algebras can be extended to generalized KacMoody algebras. For example, the Kac-Weyl formula about an irreducible representation over a Kac-Moody algebra is generalized to a formula about an irreducible representation over a generalized Kac-Moody algebra as follows.

$$
\operatorname{ch} V(\lambda)=\frac{\sum_{w \in W} \sum_{F \subseteq T, F \perp \lambda}(-1)^{l(w)+|F|} e^{w(\lambda+\rho-s(F))}}{\sum_{w \in W, F \subseteq T}(-1)^{l(w)+|F|} e^{w(\rho-s(F))}},
$$

where $T$ is the set of all imaginary simple roots, $F$ runs all over finite subsets of $T$ such that any two elements in $F$ are mutually perpendicular. We denote by $s(F)$ the sum of the roots in $F$. We call the above formula Borcherds-Kac-Weyl formula.

On the other hand, many mathematicians are interested in generalization of Hopf algebras, of which importance has been recognized in both mathematics and physics. One way to do this is to introduce a kind of weak coproduct such that $\Delta(1) \neq 1 \otimes 1$ in Ref.1. The face algebras ${ }^{[7]}$ and generalized Kac algebras ${ }^{[16]}$ are examples of this class of weak Hopf algebras. Li and Duplij have defined and studied another kind of weak Hopf algebras ${ }^{[12]}$. A bialgebra $(H, \mu, \eta, \Delta, \varepsilon)$ is called a weak Hopf algebra if there is an anti-automorphism $T$ such that $T * i d_{H} * T=i d_{H}$ and 
$i d_{H} * T * i d_{H}=T$, where $i d_{H}$ is the identity map and $*$ is the convolution product. Hopf algebras, and left of right Hopf ${ }^{[13-14]}$ algebras are weak Hopf algebras in this sense. In presented paper a weak Hopf algebra is always mean the weak Hopf algebra in this sense. The weak quantized enveloping algebras of semi-simple Lie algebras are also weak Hopf algebras ${ }^{[15]}$. Our aim is to give more nontrivial examples of weak Hopf algebras. Thanks to the definition of quantized enveloping algebra $U_{q}(\mathcal{G})$ associated a generalized Kac-Moody algebra $\mathcal{G}$ defined in Ref. 7, we can also replace the group $G\left(U_{q}(\mathcal{G})\right)$ of grouplike elements by some regular monoid as in Ref.14 and Ref. 15 , we use a new generator $J$ instead of the projector in Ref.14 and Ref.15. Our new generator $J$ satisfies $J^{m}=J$ for some integer $m \geq 2$. By this way, we obtain a subclass of bialgebra $w U_{q}(\mathcal{G})$. This bialgebra contains a sub-bialgebra $U^{\tau}(\mathcal{G})$, which is isomorphic to the a weak Hopf algebra in Ref. 15. Moreover, the quotient algebra $w U_{q}(\mathcal{G}) /(1-J)$ is isomorphic to a sub-Hopf-algebra of the quantized enveloping algebra $U_{q}(\mathcal{G})$ as Hopf algebras. As in the case of the classic quantum group $U_{q}(\mathcal{G})$, we try to determine irreducible representation of $w U_{q}(\mathcal{G})$.

Finally, let us outline the structure of this paper. In Section II, we recall some basic facts related to the quantized enveloping algebra of a generalized Kac-Moody algebra. In Section III, we give the definition of $w U_{q}(\mathcal{G})$. We study the bialgebra structure of $w U_{q}(\mathcal{G})$ in Section IV. In the final section, we study the irreducible representation of $w U_{q}(\mathcal{G})$.

\section{NOTATIONS AND PRELIMINARIES}

In this section, we fix notations and recall fundamental results about generalized Kac-Moody algebras.

Let $I=\{1, \cdots, n\}$ or the set of positive integers, and $A=\left(a_{i j}\right)_{I \times I}$, a Borcherds-Cartan matrix, i.e., it satisfies:

(1) $a_{i i}=2$ or $a_{i i} \leq 0$ for all $i \in I$,

(2) $a_{i j} \leq 0$ for all $i \neq j$,

(3) $a_{i j} \in \mathbf{Z}$,

(4) $a_{i j}=0$ if only if $a_{j i}=0$.

We say that an index $i$ is real if $a_{i i}=2$ and imaginary if $a_{i i} \leq 0$. We denote $I^{+}=\left\{i \in I \mid a_{i i}=2\right\}$ and $I^{i m}=I-I^{+}$.

Kang considered the generalized Kac-Moody algebras associated with Borcherds-Cartan matrices with charge ${ }^{[9]}$

$$
\mathbf{m}=\left\{\left(m_{i} \in \mathbf{Z}_{\geq 0}\right) \mid i \in I, m_{i}=1 \text { for } i \in I\right\}
$$

The charge $m_{i}$ is the multiplicity of the simple root corresponding to $i \in I$. In this paper, we follow [11], and assume that $m_{i}=1$ for all $i \in I$. However, we do not lose generality by this hypothesis. Indeed, if we take BorcherdsCartan matrices with some of the rows and columns identical, then the generalized Kac-Moody algebras with charge introduced in [9] can be recovered from the ones in present paper by identifying the $h_{i} s$ and $d_{i} s\left(\right.$ and hence the $\left.\alpha_{i} s\right)$ corresponding to these identical rows and columns.

Moreover, we also assume that $A$ is symmetrizable; that is, there is a diagonal matrix $D=\operatorname{diag}\left\{s_{i}>0 \mid i \in I\right\}$ such that $D A$ is a symmetric matrix. 
Let $P^{\prec}=\left(\oplus_{i \in I} \mathbf{Z} h_{i}\right) \oplus\left(\oplus_{i \in I} \mathbf{Z} d_{i}\right)$ be a free abelian group generated by the set $\left\{h_{i}, d_{i} \mid i \in I\right\}$. This free abelian group is called the co-weight lattice of $A$. The element $h_{i}$ in $\Pi=\left\{h_{i} \mid i \in I\right\}$ is called a simple co-weight. We call $\Pi$ the set of all simple co-weights. The space $\mathcal{H}=\mathbf{Q} \otimes \mathbf{z} P^{`}$ over the rational number field $\mathbf{Q}$ is said to be a Cartan subalgebra. The weight lattice is defined to be $P:=\left\{\lambda \in \mathcal{H}^{*} \mid \lambda\left(P^{y}\right) \subseteq \mathbf{Z}\right\}$, where $\mathcal{H}^{*}$ is the dual space of the Cartan subalgebra $\mathcal{H}=\mathbf{Q} \otimes_{\mathbf{z}} P^{\check{r}}$. We denote by $P^{+}$the set $\left\{\lambda \in P \mid \lambda\left(h_{i}\right) \geq 0\right.$, for every $\left.i \in I\right\}$ of dominant integral weights.

Define $\alpha_{i}, \Lambda_{i} \in \mathcal{H}^{*}$ by

$$
\begin{gathered}
\alpha_{i}\left(h_{j}\right)=a_{j i}, \quad \alpha_{i}\left(d_{j}\right)=\delta_{i j} \\
\Lambda_{i}\left(h_{j}\right)=\delta_{i j}, \quad \Lambda_{i}\left(d_{j}\right)=0 .
\end{gathered}
$$

Then $\alpha_{i}, i \in I$ are called simple roots of $A$. Let $\Pi=\left\{\alpha_{i} \mid i \in I\right\} \subset P$ be the set of simple roots. The free abelian group $Q=\oplus_{i \in I} \mathbf{Z} \alpha_{i}$ is called the root lattice. Set $Q_{+}=\sum_{i \in I} \mathbf{Z}_{\geq 0} \alpha_{i}$ and $Q_{-}=-Q_{+}$. For any $\alpha \in Q_{+}$, we can write $\alpha=\sum_{k=1}^{n} \alpha_{i_{k}}$ for $i_{1}, i_{2}, \cdots, i_{n} \in I$. We set $h t(\alpha)=n$ and call it the height of $\alpha$.

Let (.|.) be the bilinear form on $\left(\oplus_{i}\left(\mathbf{Q} \alpha_{i} \oplus \mathbf{Q} \Lambda_{i}\right)\right) \times \mathcal{H}^{*}$ defined by

$$
\left(\alpha_{i} \mid \lambda\right)=s_{i} \lambda\left(h_{i}\right),\left(\Lambda_{i} \mid \lambda\right)=s_{i} \lambda\left(d_{i}\right)
$$

Since it is symmetric on $\left(\oplus_{i}\left(\mathbf{Q} \alpha_{i} \oplus \mathbf{Q} \Lambda_{i}\right)\right) \times\left(\oplus_{i}\left(\mathbf{Q} \alpha_{i} \oplus \mathbf{Q} \Lambda_{i}\right)\right)$, one can extend this to a symmetric bilinear form on $\mathcal{H}^{*}$. Then such a form is non-degenerated.

We always assume that $\mathbf{K}$ is a field of characteristic 0 . Let $q \in \mathbf{K}$ and $q_{i}=q^{d_{i}}$. It is assumed that $q_{i} \neq \pm 1,0$ for all $i \in I$. For an indeterminant $\nu$ and an integer $m$, let

$$
[m]_{\nu}=\frac{\nu^{m}-\nu^{-m}}{\nu-\nu^{-1}},[m] !_{\nu}=[m]_{\nu} \cdots[1]_{\nu},[0]_{\nu}=1,
$$

and

$$
\left[\begin{array}{c}
m \\
s
\end{array}\right]_{\nu}=\frac{[m] !_{\nu}}{[s] !_{\nu}[m-s] !_{\nu}}
$$

Definition The quantized enveloping generalized Kac-Moody algebra $U_{q}(\mathcal{G})$ associated with a Borcherds-Cartan datum $\left(A, P^{\sim}, P, \Pi^{\sim}, \Pi\right)$ is the associated algebra with unit 1 over a field $\mathbf{K}$ of characteristic 0 , generated by the symbols $e_{i}$, $f_{i}(i \in I)$ and $P^{\sim}$ subject to the following defining relations:

$$
\begin{gathered}
q^{0}=1, q^{h} q^{h^{\prime}}=q^{h+h^{\prime}} \quad \forall h, h^{\prime} \in P, \\
q^{h} e_{i} q^{-h}=q^{\alpha_{i}(h)} e_{i}, \quad q^{h} f_{i} q^{-h}=q^{-\alpha_{i}(h)} f_{i}, \\
e_{i} f_{j}-f_{j} e_{i}=\delta_{i j} \frac{k_{i}-k_{i}^{-1}}{q_{i}-q_{i}^{-1}}, \quad \text { where } \quad k_{i}=q^{s_{i} h_{i}},
\end{gathered}
$$




$$
\begin{gathered}
\sum_{r=0}^{1-a_{i j}}(-1)^{r}\left[\begin{array}{c}
1-a_{i j} \\
r
\end{array}\right]_{i} e_{i}^{1-a_{i j}-r} e_{j} e_{i}^{r}=0 \quad \text { if } a_{i i}=2, i \neq j, \\
\sum_{r=0}^{1-a_{i j}}(-1)^{r}\left[\begin{array}{c}
1-a_{i j} \\
r
\end{array}\right]_{i} f_{i}^{1-a_{i j}-r} f_{j} f_{i}^{r}=0 \quad \text { if } \quad a_{i i}=2, i \neq j, \\
e_{i} e_{j}-e_{j} e_{i}=f_{i} f_{j}-f_{j} f_{i}=0, \quad \text { if } \quad a_{i j}=0 .
\end{gathered}
$$

The quantum generalized Kac-Moody algebra $U_{q}(\mathcal{G})$ has a Hopf algebra structure with the comultiplication $\Delta$, the counit $\varepsilon$, and antipode $S$ defined by

$$
\begin{gathered}
\Delta\left(q^{h}\right)=q^{h} \otimes q^{h}, \\
\Delta\left(e_{i}\right)=e_{i} \otimes k_{i}^{-1}+1 \otimes e_{i}, \\
\Delta\left(f_{i}\right)=k_{i} \otimes f_{i}+f_{i} \otimes 1, \\
\varepsilon\left(q^{h}\right)=1, \varepsilon\left(e_{i}\right)=\varepsilon\left(f_{i}\right)=0, \\
S\left(q^{h}\right)=q^{-h}, S\left(e_{i}\right)=-e_{i} k_{i}, S\left(f_{i}\right)=-k_{i}^{-1} f_{i}
\end{gathered}
$$

for all $h \in P^{\curlyvee}$ and $i \in I$.

Let $U_{q}^{+}(\mathcal{G})$ and $U_{q}^{-}(\mathcal{G})$ be the subalgebras of $U_{q}(\mathcal{G})$ generated by elements $e_{i}$ and $f_{i}$ respectively, for $i \in I$, and let $U_{q}^{0}(\mathcal{G})$ be the subalgebra of $U_{q}(\mathcal{G})$ generated by $q^{h}\left(h \in P^{`}\right)$. Then we have the triangular decomposition $[1,8]$

$$
U_{q}(\mathcal{G})=U_{q}^{-}(\mathcal{G}) \otimes U_{q}^{0}(\mathcal{G}) \otimes U_{q}^{+}(\mathcal{G})
$$

Finally, let us use $U_{q}^{\prime}(\mathcal{G})$ to denote a subalgebra of $U_{q}(\mathcal{G})$ generated by $e_{i}, f_{i}, q^{h}$, where $h \in \oplus_{i \in I} \mathbf{Z} s_{i} h_{i} \oplus \oplus_{i \in I} \mathbf{Z} d_{i}$. It is obvious that $U_{q}^{\prime}(\mathcal{G})$ is a Hopf algebra.

\section{WEAK QUANTUM ALGEBRAS $w U_{q}^{T}(\mathcal{G})$}

Let $m$ be a fixed positive integer. To generalize the invertibility condition $k_{i} k_{i}^{-1}=1$ in $U_{q}(\mathcal{G})$, let us introduce some new generators $J, K_{i}$ and $\bar{K}_{i}$, which subject the following relations:

$$
J^{m-1}=K_{i} \bar{K}_{i}=\bar{K}_{i} K_{i}=D_{i} \bar{D}_{i}=\bar{D}_{i} D_{i}
$$


Moreover, we assume that $J^{m}=J$ and

$$
\begin{array}{ll}
K_{i} J^{m-1}=J^{m-1} K_{i}=K_{i}, & \bar{K}_{i} J^{m-1}=J^{m-1} \bar{K}_{i}=\bar{K}_{i} . \\
D_{i} J^{m-1}=J^{m-1} D_{i}=D_{i}, & \bar{D}_{i} J^{m-1}=J^{m-1} \bar{D}_{i}=\bar{D}_{i} .
\end{array}
$$

We call an element $E_{i}$ of type one if it satisfies

$$
K_{j} E_{i}=q_{i}^{a_{i j}} E_{i} K_{j}, \quad \bar{K}_{j} E_{i}=q_{i}^{-a_{i j}} E_{i} \bar{K}_{j}
$$

Similarly, if

$$
K_{j} F_{i}=q_{i}^{-a_{i j}} F_{i} K_{j}, \quad \bar{K}_{j} F_{i}=q_{i}^{-a_{i j}} F_{i} \bar{K}_{j}
$$

then $F_{i}$ is said to be type one. Suppose

$$
K_{j} E_{i} \bar{K}_{j}=q^{a_{i j}} E_{i}
$$

Then we say that $E_{i}$ is type zero. Similarly, $F_{i}$ is type zero if it satisfies the following:

$$
K_{j} F_{i} \bar{K}_{j}=q^{-a_{i j}} F_{i}
$$

Proposition III. $1 E_{i}\left(\right.$ resp. $\left.F_{i}\right)$ is type zero if and only if $E_{i}$ is type one and $E_{i} J^{m-1}=J^{m-1} E_{i}=E_{i}$ (respectively, $\left.F_{i} J^{m-1}=J^{m-1} F_{i}=F_{i}\right)$.

Proof If $E_{i}$ is type zero, then we obtain from (III.6)

$$
K_{j} E_{i} \bar{K}_{j} K_{j}=K_{j} E_{i} J^{m-1}=q_{i}^{a_{i j}} E_{i} K_{j}
$$

On the other hand, since $J^{m-1} K_{j}=K_{j}$,

$$
K_{j} E_{i} \bar{K}_{j}=K_{j} E_{i} J^{m-1} \bar{K}_{j}=q_{i}^{a_{i j}} E_{i} K_{j} \bar{K}_{j}=q_{i}^{a_{i j}} E_{i} J^{m-1} .
$$

So $E_{i}=E_{i} J^{m-1}$. Similarly, we can prove that $E_{i}=J^{m-1} E_{i}$. Then

$$
K_{j} E_{i}=K_{j} E_{i} J^{m-1}=q_{i}^{a_{i j}} E_{i} K_{j}
$$

and

$$
E_{i} \bar{K}_{j}=J^{m-1} E_{i} \bar{K}_{j}=q_{i}^{a_{i j}} \bar{K}_{j} E_{i}
$$

That is, $E_{i}$ is type one. On the other hand, if $E_{i}$ is type one, and $E_{i} J^{m-1}=J^{m-1} E_{i}=E_{i}$, then

$$
q_{i}^{a_{i j}} E_{i}=q_{i}^{a_{i j}} E_{i} K_{j} \bar{K}_{j}=K_{j} E_{i} \bar{K}_{j}
$$


Similarly,we can prove the statement about $F_{i}$ is true. By now, we complete the proof.

The types of $E_{i}$ and $F_{i}$ are denoted by $\kappa_{i}, \kappa^{\prime}$ respectively. Let $\left.\tau=\left(\left\{\kappa_{i}\right\}_{i \in I} \mid\left\{\kappa_{i}^{\prime}\right)\right\}_{i \in I}\right)$. By now, we can give the definition the weak quantum algebra of type $\tau$ as follows:

Definition The type $\tau$ weak quantum algebra $w U_{q}^{\tau}(\mathcal{G})$ associated the generalized Kac-Moody algebra $\mathcal{G}$ is an associated algebra with unit 1 over a field $\mathbf{K}$ of characteristic 0 , generated by $J, E_{i}, F_{i}(i \in I)$ and $K_{i}, D_{i}(i \in I)$ subjecting with the following defining relations:

$$
\begin{aligned}
& 1 \neq J^{m-1}=K_{i} \bar{K}_{i}=D_{i} \bar{D}_{i}, \quad J^{m}=J, \\
& K_{i} \bar{K}_{j}=\bar{K}_{j} K_{i}, \quad K_{i} K_{j}=K_{j} K_{i}, \quad \bar{K}_{i} \bar{K}_{j}=\bar{K}_{j} \bar{K}_{i}, \\
& D_{i} \bar{D}_{j}=\bar{D}_{j} D_{i}, \quad D_{i} D_{j}=D_{j} D_{i}, \quad \bar{D}_{i} \bar{D}_{j}=\bar{D}_{j} \bar{D}_{i}, \\
& D_{i} \bar{K}_{j}=\bar{K}_{j} D_{i}, \quad K_{i} D_{j}=D_{j} K_{i}, \quad \bar{D}_{i} K_{j}=K_{j} \bar{D}_{i}, \quad \bar{D}_{i} \bar{K}_{j}=\bar{K}_{j} \bar{D}_{i}, \\
& D_{i} J=J D_{i}, \quad K_{i} J=J K_{i}, \quad \bar{K}_{i} J=J \bar{K}_{i}, \quad \bar{D}_{i} J=J \bar{D}_{i}, \\
& D_{i} J^{m-1}=D_{i}, \quad K_{i} J^{m-1}=K_{i}, \quad J^{m-1} \bar{D}_{i}=\bar{D}_{i}, \quad J^{m-1} \bar{K}_{i}=\bar{K}_{i}, \\
& E_{i} \quad F_{i} \text { are type } \tau \text {, } \\
& E_{i} F_{j}-F_{j} E_{i}=\delta_{i j} \frac{K_{i}-\bar{K}_{i}}{q_{i}-q_{i}^{-1}} \\
& \sum_{r=0}^{1-a_{i j}}(-1)^{r}\left[\begin{array}{c}
1-a_{i j} \\
r
\end{array}\right]_{i} E_{i}^{1-a_{i j}-r} E_{j} E_{i}^{r}=0 \quad \text { if } \quad a_{i i}=2, i \neq j, \\
& \sum_{r=0}^{1-a_{i j}}(-1)^{r}\left[\begin{array}{c}
1-a_{i j} \\
r
\end{array}\right]_{i} F_{i}^{1-a_{i j}-r} F_{j} F_{i}^{r}=0 \quad \text { if } \quad a_{i i}=2, i \neq j \\
& E_{i} E_{j}-E_{j} E_{i}=F_{i} F_{j}-F_{j} F_{i}=0, \quad \text { if } \quad a_{i j}=0 .
\end{aligned}
$$

If $m=1$, and the Borcherds-Cartan matrix $A$ is symmetric, then $w U_{q}^{T}(\mathcal{G})=U_{q}(\mathcal{G})$ provided that we identify $K_{i}$ with $q^{h_{i}}, \bar{K}_{i}$ with $q^{-h_{i}}, D_{i}$ with $q^{d_{i}}$ and $\bar{D}_{i}$ with $q^{-d_{i}}$. If $m=2$ and $\mathcal{G}$ is a semisimple Lie algebra, then $w U_{q}^{T}(\mathcal{G})$ has been defined and studied by Yang in Ref.[15]. Notice that the type zero was called type two by Yang. 
Lemma III.1 $J^{m-1}$ is a center idempotent element of $w U_{q}^{\tau}(\mathcal{G})$.

Proof If $E_{i}$ is type one, then $J^{m-1} E_{i}=K_{j} \bar{K}_{j} E_{i}=E_{i} K_{j} \bar{K}_{j}=E_{i} J^{m-1}$. Similarly, we can prove that $F_{i} J^{m-1}=$ $J^{m-1} F_{i}$ provided $F_{i}$ is type one. Hence this lemma follows from Proposition III.1.

In the following corollary, the subalgebra of $U_{q}(\mathcal{G})$ generated by $E_{i}, F_{i}, s_{i} h_{i}, d_{i}(i \in I)$ is denoted by $U_{q}^{\prime}(\mathcal{G})$. It is a Hopf subalgebra of $U_{q}(\mathcal{G})$.

Corollary III.1 (1) $w U_{q}^{\tau}(\mathcal{G})=w U_{q}^{\tau}(\mathcal{G}) J^{m-1} \oplus w U_{q}^{\tau}(\mathcal{G})\left(1-J^{m-1}\right)$ is a direct sum of algebras.

(2) $w U_{q}^{\tau}(\mathcal{G}) /(1-J)$ is isomorphic to the algebra $U_{q}^{\prime}(\mathcal{G})$.

(3) The subalgebra $w U_{q}^{\tau}(\mathcal{G})_{m-1}$ of $w U_{q}^{\tau}(\mathcal{G})$, which is generated by $E_{i} J^{m-1}, F_{i} J^{m-1}, K_{i}, \bar{K}_{i}, D_{i}, \bar{D}_{i}$ (i $\left.\in I\right)$, is isomorphic to the algebra $U_{q}^{\prime}(\mathcal{G})$.

(4) Let $U^{\tau}(\mathcal{G})$ be a subalgebra of $w U_{q}^{\tau}(\mathcal{G})$, which is generated by $E_{i}, F_{i}, K_{i}, \bar{K}_{i}, D_{i}, \bar{D}_{i}(i \in I)$. Suppose $\mathcal{G}$ is a semisimple Lie algebra. Then $U^{\tau}(\mathcal{G})$ is isomorphic to the algebra $\mathcal{M}_{q}^{d}(\mathcal{G})$ in [15].

Proof The proof of (1) and (2) is easy. To prove $(3)$, let us define a map $\psi$ from $U_{q}^{\prime}(\mathcal{G})$ to $w U_{q}^{\tau}(\mathcal{G}) J^{m-1}$ as follows.

$$
\psi\left(q^{s_{i} h_{i}}\right)=K_{i}, \quad \psi\left(q^{d_{i}}\right)=D_{i}, \quad \psi\left(e_{i}\right)=E_{i} J^{m-1}, \quad \psi\left(f_{i}\right)=F_{i} J^{m-1}, \quad \psi(1)=J^{m-1} .
$$

Then one can show that $\psi$ is an algebra homomorphism. Similarly we can define an algebra homomorphism a map $\varphi$ from $w U_{q}^{\tau}(\mathcal{G}) J^{m-1}$ to $U_{q}^{\prime}(\mathcal{G})$ as follows.

$$
\varphi\left(K_{i}\right)=q^{s_{i} h_{i}}, \quad \varphi\left(D_{i}\right)=q^{d_{i}}, \quad \varphi\left(E_{i} J^{m-1}\right)=e_{i}, \quad \varphi\left(F_{i} J^{m-1}\right)=f_{i}, \quad \varphi\left(J^{m-1}\right)=1 .
$$

It is easy to check that $\varphi \psi=i d$ and $\psi \varphi=i d$. This proves (3).

To prove (4), let us use $J^{\prime}$ to denote the $J$ in the algebra $\mathcal{M}_{q}^{d}(\mathcal{G})$. Then we can define a mapping $\theta$ from $U^{\tau}(\mathcal{G})$ to $\mathcal{M}_{q}^{d}(\mathcal{G})$ as follows:

$$
\theta\left(E_{i}\right)=E_{i}, \quad \theta\left(F_{i}\right)=F_{i}, \quad \theta\left(J^{m-1}\right)=J^{\prime}
$$

It is easy to prove that $\theta$ is an isomorphism.

Remark 1 By Proposition III.1, $w U_{q}^{\tau}(\mathcal{G})\left(1-J^{m-1}\right)$ is generated by $1-J^{m-1}, E_{i}\left(1-J^{m-1}\right)$ and $F_{i}\left(1-J^{m-1}\right)$, where $E_{i}$ and $F_{i}$ are type one. Since (III.15) holds in $w U_{q}^{\tau}(\mathcal{G}), E_{i}\left(1-J^{m-1}\right) F_{j}\left(1-J^{m-1}\right)=F_{j}\left(1-J^{m-1}\right) E_{i}\left(1-J^{m-1}\right)$. If all $\kappa_{i}=\bar{\kappa}_{i}=0$, then $w U_{q}^{\tau}(\mathcal{G})\left(1-J^{m-1}\right)$ is isomorphic to $\mathbf{K}$.

\section{THE BIALGEBRA STRUCTURE OF $w U_{q}^{\tau}(\mathcal{G})$}

The algebras $w U_{q}^{\tau}(\mathcal{G}) J^{m-1}$ and $w U_{q}^{\tau}(\mathcal{G})\left(1-J^{m-1}\right)$ are denoted by $w$ and $\bar{w}$ respectively in the following. By Corollary III.1, $w U_{q}^{\tau}(\mathcal{G})_{m-1}$ is isomorphic to the quantum group $U_{q}(\mathcal{G})$ provided $s_{i}=1$. Thus the comultiplication and counit of $U_{q}(\mathcal{G})$ transplant to the algebra $w U_{q}^{\tau}(\mathcal{G})_{m-1}$, and $w U_{q}^{\tau}(\mathcal{G})_{m-1}$ becomes a Hopf algebra. Moreover, we can define three maps:

$$
\Delta: w U_{q}^{\tau}(\mathcal{G}) \rightarrow w U_{q}^{\tau}(\mathcal{G}) \otimes w U_{q}^{\tau}(\mathcal{G})
$$




$$
\begin{gathered}
\varepsilon: w U_{q}^{\tau}(\mathcal{G}) \rightarrow \mathbf{K}, \\
T: w U_{q}^{\tau}(\mathcal{G}) \rightarrow w U_{q}^{\tau}(\mathcal{G}),
\end{gathered}
$$

as follows:

$$
\begin{aligned}
& \Delta\left(K_{i}\right)=K_{i} \otimes K_{i}, \quad \Delta\left(\bar{K}_{i}\right)=\bar{K}_{i} \otimes \bar{K}_{i}, \\
& \Delta\left(D_{i}\right)=D_{i} \otimes D_{i}, \quad \Delta\left(\bar{D}_{i}\right)=\bar{D}_{i} \otimes \bar{D}_{i}, \\
& \Delta(J)=J \otimes J \\
& \Delta\left(E_{i}\right)=\left\{\begin{array}{l}
1 \otimes E_{i}+E_{i} \otimes K_{i}, \quad E_{i} \quad \text { is type one } \\
J^{m-1} \otimes E_{i}+E_{i} \otimes K_{i}, \quad E_{i} \text { is type zero }
\end{array}\right. \\
& \Delta\left(F_{i}\right)=\left\{\begin{array}{l}
F_{i} \otimes 1+\bar{K}_{i} \otimes F_{i}, \quad F_{i} \quad \text { is type one } \\
F_{i} \otimes J^{m-1}+\bar{K}_{i} \otimes F_{i}, \quad F_{i} \text { is type zero },
\end{array}\right. \\
& \varepsilon\left(K_{i}\right)=\varepsilon\left(\bar{K}_{i}\right)=1, \quad \varepsilon\left(D_{i}\right)=\varepsilon\left(\bar{D}_{i}\right)=1, \quad \varepsilon(J)=1, \\
& \varepsilon\left(E_{i}\right)=\varepsilon\left(F_{i}\right)=0,
\end{aligned}
$$

while the map $T$ is defined as follows:

$$
\begin{gathered}
T(1)=1, \quad T\left(K_{i}\right)=\bar{K}_{i}, \quad T\left(\bar{K}_{i}\right)=K_{i}, \\
T(J)=J, \quad T\left(D_{i}\right)=\bar{D}_{i}, \quad T\left(\bar{D}_{i}\right)=D_{i} \\
T\left(E_{i}\right)=-E_{i} \bar{K}_{i}, \quad T\left(F_{i}\right)=-K_{i} F_{i} .
\end{gathered}
$$

Then we extend them to the whole $w U_{q}^{\tau}(\mathcal{G})$. Thus we obtain the following Lemma.

Lemma IV.1 $w U_{q}^{\tau}(\mathcal{G})$ is a bialgebra with comultiplication $\Delta$ and counit $\varepsilon$.

Proof It can be shown by direct calculation that the following relations hold.

$$
\Delta\left(K_{i}\right) \Delta\left(\bar{K}_{i}\right)=\Delta\left(\bar{K}_{i}\right) \Delta\left(K_{i}\right)=\Delta\left(J^{m-1}\right)=\Delta\left(D_{i}\right) \Delta\left(\bar{D}_{i}\right)=\Delta\left(\bar{D}_{i}\right) \Delta\left(D_{i}\right),
$$




$$
\begin{aligned}
& \Delta\left(K_{i}\right) \Delta\left(\bar{K}_{j}\right)=\Delta\left(\bar{K}_{j}\right) \Delta\left(K_{i}\right), \quad \Delta\left(D_{i}\right) \Delta\left(\bar{D}_{j}\right)=\Delta\left(\bar{D}_{j}\right) \Delta\left(D_{i}\right), \\
& \Delta\left(K_{i}\right) \Delta\left(D_{j}\right)=\Delta\left(D_{j}\right) \Delta\left(K_{i}\right), \quad \Delta\left(\bar{D}_{i}\right) \Delta\left(\bar{K}_{j}\right)=\Delta\left(\bar{D}_{i}\right) \Delta\left(\bar{K}_{j}\right), \\
& \Delta\left(K_{i}\right) \Delta\left(\bar{D}_{j}\right)=\Delta\left(\bar{D}_{j}\right) \Delta\left(K_{i}\right), \quad \Delta\left(D_{i}\right) \Delta\left(\bar{K}_{j}\right)=\Delta\left(D_{i}\right) \Delta\left(\bar{K}_{j}\right), \\
& \Delta\left(K_{i}\right) \Delta(J)=\Delta(J) \Delta\left(K_{i}\right), \quad \Delta\left(D_{i}\right) \Delta(J)=\Delta\left(D_{i}\right) \Delta(J), \\
& \Delta\left(\bar{K}_{i}\right) \Delta(J)=\Delta(J) \Delta\left(\bar{K}_{i}\right), \quad \Delta\left(\bar{D}_{i}\right) \Delta(J)=\Delta\left(\bar{D}_{i}\right) \Delta(J), \quad \Delta\left(J^{m}\right)=\Delta(J), \\
& \Delta\left(J^{m-1} K_{i}\right)=\Delta\left(K_{i}\right), \quad \Delta\left(J^{m-1} \bar{K}_{i}\right)=\Delta\left(\bar{K}_{i}\right), \\
& \Delta\left(J^{m-1} D_{i}\right)=\Delta\left(D_{i}\right), \quad \Delta\left(J^{m-1} \bar{D}_{i}\right)=\Delta\left(\bar{D}_{i}\right), \\
& \varepsilon\left(K_{i} \bar{K}_{j}\right)=\varepsilon\left(K_{i}\right) \varepsilon\left(\bar{K}_{j}\right), \quad \varepsilon\left(D_{i} \bar{D}_{j}\right)=\varepsilon\left(D_{i}\right) \varepsilon\left(\bar{D}_{j}\right), \\
& \varepsilon\left(J^{m-1} K_{i}\right)=\varepsilon\left(K_{i}\right), \quad \varepsilon\left(J^{m-1} \bar{K}_{j}\right)=\varepsilon\left(\bar{K}_{j}\right), \\
& \varepsilon\left(K_{i}\right) \varepsilon(J)=\varepsilon(J) \varepsilon\left(K_{i}\right), \quad \varepsilon\left(D_{i}\right) \varepsilon(J)=\varepsilon\left(D_{i}\right) \varepsilon(J), \\
& \varepsilon\left(\bar{K}_{i}\right) \varepsilon(J)=\varepsilon(J) \varepsilon\left(\bar{K}_{i}\right), \quad \varepsilon\left(\bar{D}_{i}\right) \varepsilon(J)=\varepsilon\left(\bar{D}_{i}\right) \varepsilon(J), \quad \varepsilon\left(J^{m}\right)=\varepsilon(J), \\
& \varepsilon\left(J^{m-1} \bar{D}_{i}\right)=\varepsilon\left(D_{i}\right), \quad \varepsilon\left(J^{m-1} \bar{D}_{j}\right)=\varepsilon\left(\bar{D}_{j}\right), \\
& \varepsilon\left(D_{i} K_{j}\right)=\varepsilon\left(K_{j} D_{i}\right), \quad \varepsilon\left(D_{i} \bar{K}_{j}\right)=\varepsilon\left(\bar{K}_{j} D_{i}\right), \quad \varepsilon\left(\bar{D}_{i} K_{j}\right)=\varepsilon\left(K_{j} \bar{D}_{i}\right) \\
& \varepsilon\left(K_{j}\right) \varepsilon\left(E_{i}\right)=q_{i}^{a_{i j}} \varepsilon\left(E_{i}\right) \varepsilon\left(K_{j}\right), \quad \varepsilon\left(F_{i}\right) \varepsilon\left(\bar{K}_{j}\right)=q_{i}^{a_{i j}} \varepsilon\left(F_{j}\right) \varepsilon\left(\bar{K}_{j}\right), \\
& \varepsilon\left(E_{i}\right) \varepsilon\left(F_{j}\right)-\varepsilon\left(F_{j}\right) \varepsilon\left(E_{i}\right)=\delta_{i j} \frac{\varepsilon\left(K_{i}\right)-\varepsilon\left(\bar{K}_{i}\right)}{q_{i}-q_{i}^{-1}} .
\end{aligned}
$$

If $E_{i}$ is type one, then

$$
\begin{aligned}
\Delta\left(K_{j}\right) \Delta\left(E_{i}\right) & =\left(K_{j} \otimes K_{j}\right)\left(\left(1 \otimes E_{i}+E_{i} \otimes K_{i}\right)\right. \\
& =K_{j} \otimes K_{j} E_{i}+K_{j} E_{i} \otimes K_{j} K_{i} \\
& =q_{i}^{a_{i j}} \Delta\left(E_{i}\right) \Delta\left(K_{j}\right) .
\end{aligned}
$$


If $E_{i}$ is type zero, then

$$
\begin{aligned}
\Delta\left(K_{j}\right) \Delta\left(E_{i}\right) \Delta\left(\bar{K}_{j}\right) & =\left(K_{j} \otimes K_{j}\right)\left(J^{m-1} \otimes E_{i}+E_{i} \otimes K_{i}\right)\left(\bar{K}_{j} \otimes \bar{K}_{j}\right) \\
& =K_{j} \bar{K}_{j} \otimes K_{j} E_{i} \bar{K}_{j}+K_{j} E_{i} \bar{K}_{j} \otimes K_{j} K_{i} \bar{K}_{j} \\
& =q_{i}^{a_{i j}} \Delta\left(E_{i}\right) .
\end{aligned}
$$

Next we prove that

$$
\Delta\left(E_{i}\right) \Delta\left(F_{j}\right)-\Delta\left(F_{j}\right) \Delta\left(E_{i}\right)=\delta_{i j} \frac{\Delta\left(K_{i}\right)-\Delta\left(\bar{K}_{i}\right)}{q_{i}-q_{i}^{-1}} .
$$

For any integers $0 \leq r, s \leq m$, if $J^{m-r} F_{j}=F_{j} J^{m-r}, J^{m-r} \bar{K}_{j}=\bar{K}_{j} J^{m-r}, J^{m-s} K_{i}=K_{i} J^{m-s}$ and $J^{m-s} E_{i}=$ $E_{i} J^{m-s}$, then

$$
\begin{aligned}
& \quad\left(J^{m-r} \otimes E_{i}+E_{i} \otimes K_{i}\right)\left(F_{j} \otimes J^{m-s}+\bar{K}_{j} \otimes F_{j}\right) \\
& \quad-\left(F_{j} \otimes J^{m-s}+\bar{K}_{j} \otimes F_{j}\right)\left(J^{m-r} \otimes E_{i}+E_{i} \otimes K_{i}\right) \\
& =J^{m-r} F_{j} \otimes E_{i} J^{m-s}+J^{m-r} \bar{K}_{j} \otimes E_{i} F_{j}+E_{i} F_{j} \otimes K_{i} J^{m-s}+E_{i} \bar{K}_{j} \otimes K_{i} F_{j} \\
& -F_{j} J^{m-r} \otimes J^{m-s} E_{i}-F_{j} E_{i} \otimes J^{m-s} K_{i}-\bar{K}_{j} J^{m-r} \otimes F_{j} E_{i}-\bar{K}_{j} E_{i} \otimes F_{j} K_{i} \\
& =J^{m-r} \bar{K}_{j} \otimes\left(E_{i} F_{j}-F_{j} E_{i}\right)+\left(E_{i} F_{j}-F_{j} E_{i}\right) \otimes J^{m-s} K_{i} .
\end{aligned}
$$

Thus (IV.11) holds for all $i, j$.

Finally, we prove that $\Delta$ satisfies the Quantum Serre relations, i.e., the relations from (III.16) to (III.18).

From $a_{i j}=0$, we obtain $E_{i} E_{j}=E_{j} E_{i}$, and $K_{i} E_{j}=q_{j}^{a_{j i}} E_{j} K_{i}=E_{j} K_{i}$. Hence

$$
\begin{aligned}
& \left(J^{m-r} \otimes E_{i}+E_{i} \otimes K_{i}\right)\left(J^{m-s} \otimes E_{j}+E_{j} \otimes K_{j}\right) \\
& \quad-\left(J^{m-s} \otimes E_{j}+E_{j} \otimes K_{j}\right)\left(J^{m-r} \otimes E_{i}+E_{i} \otimes K_{i}\right) \\
= & J^{2 m-r-s} \otimes E_{i} E_{j}+J^{m-r} E_{j} \otimes E_{i} K_{j}+E_{i} J^{m-s} \otimes K_{i} E_{j}+E_{i} E_{j} \otimes K_{i} K_{j} \\
- & J^{2 m-r-s} \otimes E_{j} E_{i}-J^{m-s} E_{i} \otimes E_{j} K_{i}-E_{j} J^{m-r} \otimes K_{j} E_{i}-E_{j} E_{i} \otimes K_{j} K_{i} \\
= & J^{m-r} E_{j} \otimes E_{i} K_{j}+E_{i} J^{m-s} \otimes K_{i} E_{j}-J^{m-s} E_{i} \otimes E_{j} K_{i}-E_{j} J^{m-r} \otimes K_{j} E_{i} \\
= & 0,
\end{aligned}
$$

for $r=1$, or $m-1$, and $s=1$ or $m-1$. So

$$
\Delta\left(E_{i}\right) \Delta\left(E_{j}\right)-\Delta\left(E_{j}\right) \Delta\left(E_{i}\right)=0
$$

Similarly, we can prove

$$
\Delta\left(F_{i}\right) \Delta\left(F_{j}\right)-\Delta\left(F_{j}\right) \Delta\left(F_{i}\right)=0 .
$$

By now we have proven that $\Delta$ satisfies the relation (III. 18).

To prove that $\Delta$ satisfies the relation (III.16), we must consider the following cases:

(1) Both $E_{i}$ and $E_{j}$ are type one.

(2) Only one of the $E_{i}$ and $E_{j}$ is type one. 
(3) Both $E_{i}$ and $E_{j}$ are type zero.

For the case (3). Since $E_{i}$ is type zero,

$$
\begin{aligned}
\Delta\left(E_{i}\right) & =J^{m-1} \otimes E_{i}+E_{i} \otimes K_{i} \\
& =\left(J^{m-1} \otimes 1\right)\left(1 \otimes E_{i}+E_{i} \otimes K_{i}\right) . \\
& =\left(1 \otimes E_{i}+E_{i} \otimes K_{i}\right)\left(J^{m-1} \otimes 1\right)
\end{aligned}
$$

Let us introduce new notations. $\left(1 \otimes E_{i}+E_{i} \otimes K_{i}\right)$ is denoted by $\Delta^{\prime}\left(E_{i}\right)$. Set $s=1-a_{i j}$. Then

$$
\begin{aligned}
& \sum_{r=0}^{s}(-1)^{r}\left[\begin{array}{l}
s \\
r
\end{array}\right] \Delta\left(E_{i}\right)^{s-r} \Delta\left(E_{j}\right) \Delta\left(E_{i}\right)^{r} \\
& =\left(J^{m-1} \otimes 1\right)^{(s+1)} \sum_{r=0}^{s}(-1)^{r}\left[\begin{array}{l}
s \\
r
\end{array}\right] \Delta^{\prime}\left(E_{i}\right)^{s-r} \Delta^{\prime}\left(E_{j}\right) \Delta^{\prime}\left(E_{i}\right)^{r} \\
& =0
\end{aligned}
$$

by the discussion in [8,pp67-68]. Hence $\Delta$ satisfies (III.16) in this case. For the other cases, the argument is more or less the same as case (3).

Similarly, we can prove that $\Delta$ satisfies(III.17). Therefore $\Delta$ and $\varepsilon$ can be extended to an algebra morphism from $w U_{q}^{\tau}(\mathcal{G})$ to $w U_{q}^{\tau}(\mathcal{G}) \otimes w U_{q}^{\tau}(\mathcal{G})$, and from $\left.w U_{q}^{\tau} \mathcal{G}\right)$ to $\mathbf{K}$, respectively.

It is easy to prove that

$$
\begin{gathered}
(\Delta \otimes 1) \Delta(X)=(1 \otimes \Delta) \Delta(X), \\
(\varepsilon \otimes 1) \Delta(X)=(1 \otimes \varepsilon) \Delta(X)
\end{gathered}
$$

for any $X=E_{i}, F_{i}, K_{i}, \bar{K}_{i}, D_{i}, \bar{D}_{i}, J$. Since $\Delta, \varepsilon$ are algebra morphisms, (IV.12) and (IV.13) hold for any $X \in w U_{q}^{\tau}(\mathcal{G})$. By now we have completed the proof.

Next we prove that the map T, defined by (IV.8),(IV.9),(IV.10), is a weak antipode of the subbialgebra of the bialgebra $w U_{q}^{\tau}(\mathcal{G})$ generated by $E_{i}, F_{i}, K_{i}, \bar{K}_{i}, D_{i}, \bar{D}_{i}, J^{m-1}$. First we prove that $T$ can be extended to an antiautomorphism of $w U_{q}^{\tau}(\mathcal{G})$. It is easy to prove the following relations are true.

$$
\begin{gathered}
T\left(K_{i}\right) T\left(\bar{K}_{j}\right)=T\left(\bar{K}_{j}\right) T\left(K_{i}\right), \quad T\left(D_{i}\right) T\left(\bar{D}_{j}\right)=T\left(\bar{D}_{j}\right) T\left(D_{i}\right), \\
T\left(D_{i}\right) T\left(\bar{K}_{j}\right)=T\left(\bar{K}_{j}\right) T\left(D_{i}\right), \quad T\left(K_{i}\right) T\left(\bar{D}_{j}\right)=T\left(\bar{D}_{j}\right) T\left(K_{i}\right), \\
T\left(\bar{D}_{i}\right) T\left(\bar{K}_{j}\right)=T\left(\bar{K}_{j}\right) T\left(\bar{D}_{i}\right), \quad T\left(J^{m-1}\right) T\left(\bar{K}_{i}\right)=T\left(\bar{K}_{i}\right) \quad T\left(J^{m-1}\right) T\left(K_{i}\right)=T\left(K_{i}\right), \\
T\left(K_{i} J\right)=T(J) T\left(K_{i}\right)=T\left(J K_{i}\right), \quad T\left(D_{i} J\right)=T\left(D_{i}\right) T(J)=T\left(J D_{i}\right), \\
T\left(\bar{K}_{i} J\right)=T(J) T\left(\bar{K}_{i}\right)=T\left(J \bar{K}_{i}\right), \quad T\left(\bar{D}_{i} J\right)=T\left(\bar{D}_{i}\right) T(J)=T\left(J \bar{D}_{i}\right), \quad T\left(J^{m}\right)=T(J),
\end{gathered}
$$




$$
\begin{gathered}
T\left(J^{m-1}\right) T\left(\bar{D}_{i}\right)=T\left(\bar{D}_{i}\right) \quad T\left(J^{m-1}\right) T\left(D_{i}\right)=T\left(D_{i}\right) . \\
T\left(E_{i}\right) T\left(E_{j}\right)=T\left(E_{j}\right) T\left(E_{i}\right), \quad T\left(F_{i}\right) T\left(F_{j}\right)=T\left(F_{j}\right) T\left(F_{i}\right), \quad \text { if } \quad a_{i j}=0 .
\end{gathered}
$$

If $E_{i}$ is type one, then

$$
T\left(E_{i}\right) T\left(K_{j}\right)=-E_{i} \bar{K}_{i} \bar{K}_{j}=-q_{i}^{a_{i j}} \bar{K}_{j} E_{i} \bar{K}_{i}=q_{i}^{a_{i j}} T\left(K_{j}\right) T\left(E_{i}\right) .
$$

If $E_{i}$ is type zero, then

$$
T\left(\bar{K}_{j}\right) T\left(E_{i}\right) T\left(K_{j}\right)=-K_{j} E_{i} K_{i} \bar{K}_{j}=-q_{i}^{a_{i j}} E_{i} K_{i}=q_{i}^{a_{i j}} T\left(E_{i}\right) .
$$

Similarly, we can prove

$$
T\left(F_{i}\right) T\left(K_{j}\right)=q_{i}^{-a_{i j}} T\left(K_{j}\right) T\left(F_{i}\right)
$$

if $F_{i}$ is type one, and

$$
T\left(\bar{K}_{j}\right) T\left(F_{i}\right) T\left(K_{j}\right)=q_{i}^{-a_{i j}} T\left(F_{i}\right)
$$

if $F_{i}$ is type zero. Moreover,

$$
\begin{aligned}
T\left(F_{j}\right) T\left(E_{i}\right)-T\left(E_{i}\right) T\left(F_{j}\right) & =K_{j}\left(F_{j} E_{i}\right) \bar{K}_{i}-E_{i} \bar{K}_{i} K_{j} F_{j} \\
& =q_{j}^{-a_{j j}} q_{i}^{a_{i j}} F_{j} E_{i} K_{j} \bar{K}_{i}-q_{j}^{-a_{j j}} q_{j}^{a_{j i}} F_{j} E_{i} K_{j} \bar{K}_{i} \\
& =\delta_{i j} \frac{\bar{K}_{i}-K_{i}}{q_{i}-q_{i}^{-1}} K_{j} \bar{K}_{i} \\
& =\delta_{i j} \frac{T\left(K_{i}\right)-T\left(\bar{K}_{i}\right)}{q_{i}-q_{i}^{-1}}
\end{aligned}
$$

Similarly to $[15, \mathrm{p} .8]$, we can prove the following anti-relations to the quantum Serre relations hold.

$$
\begin{aligned}
& \sum_{r=0}^{s}(-1)^{r}\left[\begin{array}{l}
s \\
r
\end{array}\right] T\left(E_{i}\right)^{r} T\left(E_{j}\right) T\left(E_{i}\right)^{s-r}=0, \quad \text { if } \quad a_{i i}=2, \\
& \sum_{r=0}^{s}(-1)^{r}\left[\begin{array}{l}
s \\
r
\end{array}\right] T\left(F_{i}\right)^{r} T\left(F_{j}\right) T\left(F_{i}\right)^{s-r}=0 \quad \text { if } \quad a_{i i}=2,
\end{aligned}
$$

where $s=1-a_{i j}$.

From the above discussion, we get that $T$ is an anti-automorphism of $w U_{q}^{\tau}(\mathcal{G})$. Let $U^{\tau}$ be a subalgebra of $w U_{q}^{\tau}(\mathcal{G})$ generated by $K_{i}, \bar{K}_{i}, D_{i}, \bar{D}_{i}, E_{i}, F_{i}, J^{m-1}$.

Theorem IV.2 $T$ is a weak antipode of $U^{\tau}(\mathcal{G})$ and $U^{\tau}(\mathcal{G})$ is a weak Hopf algebra.

Proof It is easy to verified the following relations hold

$$
(i d * T * i d)(X)=X
$$




$$
(T * i d * T)(X)=T(X),
$$

for $X=K_{i}, \bar{K}_{i}, D_{i}, \bar{D}_{i}, E_{i}, F_{i}, J^{m-1}$.

Since

$$
i d * T * i d=(\mu \otimes 1) \mu(i d \otimes T \otimes i d)(\Delta \otimes 1) \Delta,
$$

$i d * T * i d$ is a linear automorphism of $w U_{q}^{\tau}(\mathcal{G})$. To prove $(i d * T * i d)(X)=X$, for any $X \in U^{\tau}(\mathcal{G})$, we only need prove that

$$
(i d * T * i d)(x y)=x y
$$

provided that $(i d * T * i d)(x)=x$, and $y$ is one of the generators $K_{i}, \bar{K}_{i}, D_{i}, \bar{D}_{i}, E_{i}, F_{i}, J^{m-1}$. Suppose $(\Delta \otimes 1) \Delta(x)=$ $\sum x_{(1)} \otimes x_{(2)} \otimes x_{(3)}$. Then $(\Delta \otimes 1) \Delta\left(x J^{m-1}\right)=\sum x_{(1)} J^{m-1} \otimes x_{(2)} J^{m-1} \otimes x_{(3)} J^{m-1}$ and hence

$$
i d * T * i d\left(x J^{m-1}\right)=\sum x_{(1)} T\left(x_{(2)}\right) x_{(3)} J^{m-1}=x J^{m-1} .
$$

Similarly

$$
\begin{aligned}
i d * T * i d\left(x E_{i}\right)= & \sum x_{(1)} J^{m-1} T\left(x_{(2)} J^{m-1}\right) x_{(3)} E_{i}+\sum x_{(1)} J^{m-1} T\left(x_{(2)} E_{i}\right) x_{(3)} K_{i}+ \\
& \sum x_{(1)} E_{i} T\left(x_{(2)} K_{i}\right) x_{(3)} K_{i} \\
= & \sum x_{(1)} T\left(x_{(2)}\right) x_{(3)} E_{i}-\sum x_{(1)} E_{i} \bar{K}_{i} T\left(x_{(2)}\right) x_{(3)} K_{i}+ \\
& \sum x_{(1)} E_{i} \bar{K}_{i} T\left(x_{(2)}\right) x_{(3)} K_{i} \\
= & x E_{i},
\end{aligned}
$$

if $E_{i}$ is type zero. We can prove (IV.14) is true for other generators of $U^{\tau}(\mathcal{G})$. So $i d * T * i d(x)=x$ for any $x \in U^{\tau}(\mathcal{G})$ by induction.

Similarly, we can prove $T * i d * T(x)=T(x)$ for any $x \in U^{\tau}(\mathcal{G})$. So $T$ is a weak antipode of $U^{\tau}(\mathcal{G})$, and $U^{\tau}(\mathcal{G})$ is a weak Hopf algebra.

Corollary IV.1 $w U_{q}^{\tau}(\mathcal{G})$ is a weak Hopf algebra if and only if $m=2,3$. Moreover, if $m=2,3$, then $w U_{q}^{\tau}(\mathcal{G})$ is a noncommutative and noncocommutative weak Hopf algebra with the weak antipode T, but not a Hopf algebra.

Proof Since $m=2, J^{2}=J$. Then

$$
i d * T * i d(J)=J^{3}=J=T(J)=T * i d * T(J) .
$$

Similarly, if $m=3$, then $J^{3}=J$. Thus $w U_{q}^{\tau}(\mathcal{G})$ is a weak Hopf algebra provided that $m=2,3$.

If $w U_{q}^{\tau}(\mathcal{G})$ is a weak Hopf algebra, then $i d * T * i d(J)=J^{3}=J$. From this and $J^{m}=J$, we can obtain either $J^{2}=J$ or $J^{3}=J$. Thus $m=2,3$ by our assumption.

Suppose $w U_{q}^{\tau}(\mathcal{G})$ is a Hopf algebra with antipode $S$. Then $S(J) J=1$. On the other hand, since $J^{m}=J$, $\left(1-J^{m-1}\right) J=0$ implies that $J^{m-1}=1$. This is contradict to our assumption. So $w U_{q}^{\tau}(\mathcal{G})$ is not a Hopf algebra. 
Corollary IV.2 $U^{\tau}(\mathcal{G})$ is a noncommutative and noncocommutative weak Hopf algebra with the weak antipode $T$, but not a Hopf algebra. Moreover, $U^{\tau}(\mathcal{G}) J^{m-1}$ is isomorphic to $U_{q}^{\prime}(\mathcal{G})$ as Hopf algebras.

Proof It follows from Corollary III.1, Theorem IV.2.

Proposition IV.1 Suppose $m \geq 4$ and $w U_{q}^{\tau}(\mathcal{G})$ has a subalgebra $W$ containing $J^{r}$ for some $1 \leq r \leq m-1$. If $W$ is a weak Hopf algebra, then

$$
r=\left\{\begin{array}{l}
m-1, \quad \text { if } m \quad \text { is even, } \\
\frac{1}{2}(m-1), \quad \text { if } m \text { is odd. }
\end{array}\right.
$$

Proof Since $W$ is a weak Hopf algebra, $J^{r}=T * i d * T(J)=J^{3 r}$. Hence $J^{3 r}=J^{r}$ and $2 r=k(m-1)$ for some integer number $k$. If $m$ is even, $k=2$, then $r=m-1$. If $m$ is odd, then $k=1,2$, and $r=m-1, \frac{1}{2}(m-1)$ respectively.

To simplify notations, let us use $\mathcal{B}_{m}$ to denote the bialgebra $w U_{q}^{\tau}(\mathcal{G})$, where $m$ is the minimal integer number satisfying $J^{m}=J$.

Proposition IV.2 Suppose $k=2, r=\frac{1}{2}(m-1)$. The subalgebra $W_{3}$ of $w U_{q}^{\tau}(\mathcal{G})$ generated by $E_{i}, F_{i}, K_{i}, \bar{K}_{i}, D_{i}, \bar{D}_{i}, J^{r}$ for $i \in I$ is isomorphic to $\mathcal{B}_{3}$.

Suppose $k=1, r=m-1$. The subalgebra $W_{2}$ of $w U_{q}^{\tau}(\mathcal{G})$ generated by $E_{i}, F_{i}, K_{i}, \bar{K}_{i}, D_{i}, \bar{D}_{i}, J^{r}$ for $i \in I$ is isomorphic to $\mathcal{B}_{2}$.

Proof If $k=1$, and $r=m-1$, then $W_{2}$ is isomorphic to $\mathcal{B}_{2}$ by Corollary IV.2.

If $m$ is odd, $k=2$, and $r=\frac{1}{2}(m-1)$, then we can prove that $W_{3}$ is isomorphic to $\mathcal{B}_{3}$ similarly.

Let $H$ be a coalgebra. The set of group-like elements of $H$ is denoted by $G(H)$ in the next proposition.

Proposition IV.3 $G\left(U^{\tau}(\mathcal{G})\right)=G\left(U^{\tau}(\mathcal{G}) J^{m-1}\right) \cup\{1\}$.

Proof If $g \in G\left(U^{\tau}(\mathcal{G})\right)$, then $g=g J^{m-1}+g\left(1-J^{m-1}\right)$. Let $g_{1}=g J^{m-1}, g_{2}=g\left(1-J^{m-1}\right)$. Then $g \otimes g=\Delta(g)=$ $g_{1} \otimes g_{1}+g_{1} \otimes g_{2}+g_{2} \otimes g_{1}+g_{2} \otimes g_{2}$. Since $\Delta\left(g_{1}\right)=g_{1} \otimes g_{1}$ is a group-like element, $\Delta\left(g_{2}\right)=g_{1} \otimes g_{2}+g_{2} \otimes g_{1}+g_{2} \otimes g_{2}$. So

$$
\begin{aligned}
(1 \otimes \Delta) \Delta\left(g_{2}\right) & =g_{1} \otimes g_{1} \otimes g_{2}+g_{1} \otimes g_{2} \otimes g_{1} \\
& +g_{1} \otimes g_{2} \otimes g_{2}+g_{2} \otimes g_{1} \otimes g_{1} \\
& +g_{2} \otimes g_{1} \otimes g_{2}+g_{2} \otimes g_{2} \otimes g_{1} \\
& +g_{2} \otimes g_{2} \otimes g_{2} .
\end{aligned}
$$

Then

$$
\left\{\begin{array}{l}
(T * i d * T)\left(g_{2}\right)=T\left(g_{2}\right) g_{2} T\left(g_{2}\right)=T\left(g_{2}\right), \\
(i d * T * i d)\left(g_{2}\right)=g_{2} T\left(g_{2}\right) g_{2}=g_{2} .
\end{array}\right.
$$

Because $U^{\tau}(\mathcal{G})\left(1-J^{m-1}\right)$ is generated by $E_{i}\left(1-J^{m-1}\right), F_{j}\left(1-J^{m-1}\right), 1-J^{m-1}$ and $T\left(E_{i}\left(1-J^{m-1}\right)\right)=T\left(F_{j}(1-\right.$ $\left.\left.J^{m-1}\right)\right)=0, T\left(g_{2}\right)=k\left(1-J^{m-1}\right)$ for some $k \in \mathbf{K}$. From (IV.16), we obtain the following:

$$
\left\{\begin{array}{l}
k^{2} g_{2}=k^{2}\left(1-J^{m-1}\right)^{2} g_{2}=k\left(1-J^{m-1}\right), \\
k g_{2}^{2}\left(1-J^{m-1}\right)=k g_{2}^{2}=g_{2} .
\end{array}\right.
$$


If $k=0$, then $g=g_{1} \in G\left(U^{\tau}(\mathcal{G})\right)$. If $k \neq 0$, then $g_{2}=\frac{1}{k}\left(1-J^{m-1}\right)$. Thus

$$
\frac{1}{k}\left(1 \otimes 1-J^{m-1} \otimes J^{m-1}\right)=\frac{1}{k} g_{1} \otimes\left(1-J^{m-1}\right)+\frac{1}{k}\left(1-J^{m-1}\right) \otimes g_{1}+\frac{1}{k^{2}}\left(1-J^{m-1}\right) \otimes\left(1-J^{m-1}\right) .
$$

Multiplying by $k\left(J^{m-1} \otimes 1\right)$ on the both sides of the above equation, we get

$$
J^{m-1} \otimes 1-J^{m-1} \otimes J^{m-1}=g_{1} \otimes\left(1-J^{m-1}\right) .
$$

Similarly, we have

$$
1 \otimes J^{m-1}-J^{m-1} \otimes J^{m-1}=\left(1-J^{m-1}\right) \otimes g_{1}
$$

Then

$$
1 \otimes 1-J^{m-1} \otimes J^{m-1}=J^{m-1} \otimes 1+1 \otimes J^{m-1}-2 J^{m-1} \otimes J^{m-1}+\frac{1}{k}\left(1-J^{m-1}\right) \otimes\left(1-J^{m-1}\right) .
$$

Hence

$$
\left(1-J^{m-1}\right) \otimes\left(1-J^{m-1}\right)=\frac{1}{k}\left(1-J^{m-1}\right) \otimes\left(1-J^{m-1}\right)
$$

Consequently, $k=1$. Notice that the set of group-like elements of $U^{\tau}(\mathcal{G}) J^{m-1}$ is a monoid generated by $K_{i}, \bar{K}_{i}, D_{i}, \bar{D}_{i}$ and $J^{m-1}$, and the elements from this monoid are linearly independent over $\mathbf{K}$. So we get $g_{1}=J^{m-1}$ from $1 \otimes J^{m-1}-$ $J^{m-1} \otimes J^{m-1}=\left(1-J^{m-1}\right) \otimes J^{m-1}=\left(1-J^{m-1}\right) \otimes g_{1}$. Hence $g=1$.

Proposition IV.4 Suppose $\varphi$ is an automorphism of the bi-algebra $w U_{q}^{\tau}(\mathcal{G})$. Then $\varphi(J)=J^{r}$ for some $1 \leq r \leq m-1$ only if there exists $1 \leq s \leq m-1$ such that $r s \equiv 1 \bmod (m-1)$. Moreover $\varphi\left(J^{m-1}\right)=J^{m-1}$ and the restriction of $\varphi$ on $w($ resp. $\bar{w})$ is an isomorphism of $w$ (respect. $\bar{w})$.

Proof From $J^{m}=J$, we obtain $\varphi(J)^{m}=\varphi(J)$. Thus $\varphi(J)^{m} J^{m-1}=\varphi(J) J^{m-1}$ is a group-like element in $w U_{q}^{\tau}(\mathcal{G}) \subseteq$ $U_{q}(\mathcal{G})$. Suppose $\varphi(J)=\varphi(J) J^{m-1}+x$, where $x=\varphi(J)\left(1-J^{m-1}\right) \in \bar{w}$. Since $T \varphi(J)=\varphi(T(J))=\varphi(J), T x=x$. Consequently, $x=k\left(1-J^{m-1}\right)$. Then

$$
\begin{aligned}
\Delta(\varphi(J)) & =\left(\varphi(J) J^{m-1}+x\right) \otimes\left(\varphi(J) J^{m-1}+x\right) \\
& =\varphi(J) J^{m-1} \otimes \varphi(J) J^{m-1}+k\left(1 \otimes 1-J^{m-1} \otimes J^{m-1}\right) \\
& =\varphi(J) J^{m-1} \otimes \varphi(J) J^{m-1}+k \varphi(J) J^{m-1} \otimes\left(1-J^{m-1}\right) \\
& +k\left(1-J^{m-1}\right) \otimes \varphi(J) J^{m-1}
\end{aligned}
$$

From (IV.18), we obtain that

$$
k\left(1 \otimes 1-J^{m-1} \otimes J^{m-1}\right)=k \varphi(J) J^{m-1} \otimes\left(1-J^{m-1}\right)+k\left(1-J^{m-1}\right) \otimes \varphi(J) J^{m-1} .
$$

Hence

$$
k\left(1-J^{m-1}\right)=k \varphi(J) J^{m-1}\left(1-J^{m-1}\right)+k\left(1-J^{m-1}\right) \varphi(J) J^{m-1}=0 .
$$


So $k=0$ and $\varphi(J)=\varphi(J) J^{m-1}$. Notice that the monoid of all group-like elements of $w U_{q}^{\tau}(\mathcal{G})$ is generated by $K_{i}, D_{i}, \bar{K}_{i}, \bar{D}_{i}$ and $J$. Since $\varphi\left(J^{m}\right)=\varphi(J)^{m}=\varphi(J) \varphi(J)=J^{r}$ for some $1 \leq r \leq m-1$. Consequently $\varphi\left(J^{m-1}\right)=$ $J^{m-1}$, and the restriction of $\varphi$ on $w$ is a Hopf algebra isomorphism of $w$. Similarly, we can prove the restriction of $\varphi$ on $\bar{w}$ is an algebra isomorphism of $\bar{w}$. Since $\varphi$ is an isomorphism, there exists $s$ such that $1 \leq s \leq m-1$ and

$$
\varphi\left(J^{s}\right)=J^{r s}=J
$$

Thus $r s \equiv 1 \bmod (m-1)$.

Remark If $r, s$ satisfy $r s \equiv 1 \bmod (m-1)$, then it is easy to prove that the mapping $\varphi$ defined as follows is an automorphism of the bialgebra $w U_{q}^{\tau}(\mathcal{G}): \varphi\left(E_{i}\right)=E_{i}, \varphi\left(F_{i}\right)=F_{i}, \varphi\left(K_{i}\right)=K_{i}, \varphi\left(\bar{K}_{i}\right)=\bar{K}_{i}, \varphi\left(D_{i}\right)=D_{i}, \varphi\left(\bar{D}_{i}\right)=$ $\bar{D}_{i}, \varphi(J)=J^{r}$. The inverse mapping $\psi$ of $\varphi$ is given by the following: $\psi\left(E_{i}\right)=E_{i}, \psi\left(F_{i}\right)=F_{i}, \psi\left(K_{i}\right)=K_{i}, \psi\left(\bar{K}_{i}\right)=$ $\bar{K}_{i}, \psi\left(D_{i}\right)=D_{i}, \psi\left(\bar{D}_{i}\right)=\bar{D}_{i}, \psi(J)=J^{s}$.

If the equation $r s \equiv 1 \bmod (m-1)$ has only trivial solution $r=s=1$, for example, $m-1$ is a prime, then we can prove the following:

Corollary IV.3 Suppose $\mathcal{G}$ is a semi-simple Lie algebra, and the equation $r s \equiv 1 \bmod (m-1)$ has trivial solution $r=s=1$. Then the automorphism group of the bialgebra of $w U_{q}^{\tau}(\mathcal{G})$ is the semi-direct product of $N$ and $H$, where $H$ is the group of diagram automorphism, and $N$ is the group of diagonal automorphism and it is a normal subgroup of the automorphism group of $w U_{q}^{\tau}(\mathcal{G})$.

Proof Similar to the proof of Theorem 5.1 in Ref.15.

Remark (1) If $\mathcal{G}$ is a semisimple Lie algebra, then $U^{\tau}(\mathcal{G})$ is isomorphic to the weak quantum algebra defined by Yang in Ref. [15].

(2) Let $\mathcal{G}$ be a generated Kac-Moody algebra determined by a Borcherds matrix A. Suppose $I^{i m} \neq \phi$. Then $\varphi_{s}$ for $1<s<m-1$ are automorphisms of $w U_{q}^{\tau}(\mathcal{G})$, where $\varphi_{s}$ defined as follows: $\varphi_{s}\left(E_{i}\right)=E_{i}, \varphi_{s}\left(F_{i}\right)=F_{i}, \varphi_{s}\left(K_{i}\right)=$ $K_{i}, \varphi_{s}\left(\bar{K}_{i}\right)=\bar{K}_{i}, \varphi_{s}\left(D_{i}\right)=J^{m-1-s} D_{i}, \varphi_{s}\left(\bar{D}_{i}\right)=J^{s} \bar{D}_{i}$ for any $i \in I$.

\section{THE REPRESENTATIONS OF $w U_{q}^{\tau}(\mathcal{G})$}

In this section, we try to determine the irreducible representations of $w U_{q}^{\tau}(\mathcal{G})$. Suppose $m-1$ is invertible in the field $\mathbf{K}$ in this section. Then

$$
e=\frac{1}{m-1} \sum_{r=1}^{m-1} J^{r}
$$

is a well-defined element. It is easy to verify that $e^{2}=e$ and $J^{m-1} e=e$. Thus $e \in w$.

Suppose $V$ is a simple module over the bi-algebra $w U_{q}^{\tau}(\mathcal{G})$. Then $V=J^{m-1} V \oplus\left(1-J^{m-1}\right) V$ is a direct sum of $w U_{q}^{\tau}(\mathcal{G})$ modules. So either $V=J^{m-1} V$, or $V=\left(1-J^{m-1}\right) V$.

If $V=\left(1-J^{m-1}\right) V$, then $J v=0$ for any $v \in V$, and $\bar{K}_{i} v=\bar{K}_{i} J^{m} v=0$ for any $v \in V$. 
If $V=J^{m-1} V$, then $J^{m-1} v=v$ for any $v \in V$. Suppose $v \in V$ satisfying $K_{i} v=\lambda_{i} v$, then $\lambda_{i} \neq 0$. In this case, $K_{i} \bar{K}_{i} K_{i} v=\lambda_{i}^{2} \bar{K}_{i} v=\lambda_{i} v$. So $\bar{K}_{i} v=\frac{1}{\lambda_{i}} v$.

By now we have completed the proof of the following proposition.

Proposition $V .1$ Let $V$ be simple $w U_{q}^{\tau}(\mathcal{G})$-module. Then either $J^{m-1} v=v$ for all $v \in V$, or $J^{m-1} v=0$ for any $v \in V$. Suppose there exists an $i \in I$ such that $K_{i} v=\lambda_{i} v$ for some nonzero vector $v$. Then $\bar{K}_{i} v=\bar{\lambda}_{i} v$ for $\lambda_{i}$, where $\bar{\lambda}_{i}=\left\{\begin{array}{ll}\lambda_{i}^{-1} & \text { if } \lambda_{i} \neq 0 ; \\ 0 & \text { if } \lambda_{i}=0 .\end{array}\right.$ Moreover, $\lambda_{i} \neq 0$ if and only if $J^{m-1} v=v$.

Let $V=J^{m-1} V$. Suppose $\left(F_{i} e-e F_{i}\right) V=\left(E_{i} e-e E_{i}\right) V=0$ for any $i \in I$, then $\mathrm{eV}$ is a module over $w U_{q}^{\tau}(\mathcal{G})$. Hence $V=e V \oplus\left(e-J^{m}\right) V$ is a sum of $w U_{q}^{\tau}(\mathcal{G})$ modules. So either $V=e V$, or $V=\left(e-J^{m-1}\right) V$.

If $V=e V$, then $J v=v$ for any $v \in V$. So $V$ can be viewed as a module over $w U_{q}^{\tau}(\mathcal{G}) /(1-J)$. Notice that $w U_{q}^{\tau}(\mathcal{G}) /(1-J)$ is isomorphic to $U_{q}^{\prime}(\mathcal{G})$ by Corollary III.1(2). In this case, $V$ has been studied by Kang ${ }^{[9]}$. For example, the limit of highest weight simple module is a highest weight simple module over the generalized Kac-Moody algebra $\mathcal{G}$ with the same weight $\lambda$. Then this simple module is unique determined by its formally Borcherd-Kac-Weyl character formula ( see Section I).

If $V=\left(e-J^{m-1}\right) V$, then $e v=0$ for any $v \in V$. Suppose a nonzero element $v \in V$ satisfies $K_{i} v=\lambda v$ and $J v=\gamma v$. Then $\gamma+\cdots+\gamma^{m-1}=0$. So $\gamma^{m-1}=1$ and $\gamma$ is a primitive $(m-1)$-th root of 1 .

Suppose $V=\left(1-J^{m-1}\right) V$ and $\mathbf{K}$ is an algebraically closed field. Then $J V=0$ and $K_{i} V=\bar{K}_{i} V=0$ for any $i \in I$ by Proposition V.1. Similarly, we can prove that $D_{i} V=\bar{D}_{i} V=0$ for all $i \in I$. Hence $E_{i} F_{j} V=F_{j} E_{i} V$ for all $i, j \in I$. Moreover, $V$ can be viewed as a module over $\bar{w}$. Recall that $\bar{w}$ is generated by $1-J^{m-1}, E_{i}\left(1-J^{m-1}\right)$, and $F_{j}\left(1-J^{m-1}\right)$, where $E_{i}, F_{j}$ are type one. Hence $E_{i}\left(1-J^{m-1}\right) F_{j}\left(1-J^{m-1}\right) V=F_{j}\left(1-J^{m-1}\right) E_{i}\left(1-J^{m-1}\right) V$ for all $i, j \in I$. In the following, we try to determine the structure of $V$ in some special case.

Let $X_{i}=E_{i}\left(1-J^{m}\right), Y_{i}=F_{i}\left(1-J^{m}\right)$. Then every simple module $V$ over $\bar{w}$ is a module over the algebra generated by $\left\{X_{i}, Y_{j} \mid\right.$ for $\left.i \in I_{1}, J \in I_{2}\right\}$, where $I_{1}=\left\{i \in I \mid E_{i}\right.$ is type one $\}, I_{2}=\left\{j \in I \mid F_{j}\right.$ is type one $\}$. The generators $X_{i}, Y_{j}$ satisfy the following relation:

$$
\begin{gathered}
X_{i} Y_{j}=Y_{j} X_{i}, \\
\sum_{r=0}^{1-a_{i j}}(-1)^{r}\left[\begin{array}{c}
1-a_{i j} \\
r
\end{array}\right]_{i} X_{i}^{1-a_{i j}-r} X_{j} X_{i}^{r}=0 \quad \text { if } \quad a_{i i}=2, i \neq j, \\
\sum_{r=0}^{1-a_{i j}}(-1)^{r}\left[\begin{array}{c}
1-a_{i j} \\
r
\end{array}\right]_{i} Y_{i}^{1-a_{i j}-r} Y_{j} Y_{i}^{r}=0 \quad \text { if } \quad a_{i i}=2, i \neq j, \\
X_{i} X_{j}-X_{j} X_{i}=Y_{i} Y_{j}-Y_{j} Y_{i}=0, \quad \text { if } \quad a_{i j}=0 .
\end{gathered}
$$

This simple module $V$ satisfies $J V=0$. From the above discussion, we obtain the following result.

Corollary $V .1$ If $a_{i j}=0$ for any $i \in\left(I_{1} \cup I_{2}\right) \cap I^{+}$, then every simple module over $\bar{w}$ is isomorphic to $\bar{w} / M$, where $M$ is a maximal ideal of $\bar{w}$. 
By Corollary V.1, the only simple over $\bar{w}$ is $\mathbf{K}[x] /(p(x))$ if $\left|I_{1} \cup I_{2}\right|=1$, where $p(x)$ is an irreducible polynomial in $\mathbf{K}[x]$. Suppose $\mathbf{K}$ is an algebraically closed field. If $a_{i j}=0$ for any $i \in\left(I_{1} \cup I_{2}\right) \cap I^{+}$, and $\left|I_{1} \cup I_{2}\right|=n$, then the simple module $V$ over $\bar{w}$ is isomorphic to $\mathbf{K}\left[X_{i}, Y_{j} \mid i \in I_{1}, J_{j} \in I_{2}\right] /\left(\left\{X_{i}-a_{i}, Y_{j}-b_{j} \mid i \in I_{1}, j \in I_{2}\right\}\right)$ for some $\left(\left(a_{i}\right)_{i \in I_{1}},\left(b_{j}\right)_{j \in I_{2}}\right) \in \mathbf{K}^{n}$.

\section{ACKNOWLEDGMENT}

The author is sponsored by SRF for ROCS,SEM. He is also sponsored by the Scientific Research Foundation of Zhejiang Provincial Education Department. The author would like thank the referee for his/her suggestion and help.

* Electronic address: wzx@zju.edu.cn

1 Aizawwa, N. and Isaac, P.S., Weak Hopf algebras corresponding to $U_{q}\left[s l_{n}\right]$, J. Math. Phys. 44, 5250-5267(2003).

2 Böhm,G.,Nil,F., and Szlachányi,K. Weak Hopf algebras I,Integral theory and $C^{*}$-structure, J.Algebra,221(1998),385-438.

${ }^{3}$ R.E. Borcherds, Generalised Kac-Moody algebras, J. Algebra 115(1988), 501-512.

4 R.E. Borcherds, Monstrous moonshine and monstrous Lie superalgebras, Invent. Math., 109(1992), 405-444.

${ }^{5}$ R.E. Borcherds, Vertex algebras, Kac-Moody algebras and the monster,Proc. Nat. Acad. Sci. USA,83(1986),3068-3071.

${ }^{6}$ Chin,W. and Musson,I.M., Corrigenda, the coradical filtration for quantized enveloping algebras, J. Lond.Math. Soc. 61(2000),319-320.

${ }^{7}$ Hayashi,T., An algebra related to the fusion rules of Wess-Zumino-Witten models, Lett. Math. Phs. 22, 291-296(1991)

8 Jantzen, J.C., Lectures on Quantum Group (American Mathematical Society, Providence, RI, 1995) vol.6.

9 S.-J.Kang, Quantum deformations of generalised Kac-Moody algebras and their modules, J. of Algebra 175(1995),1041-1066.

10 S.J.Kang, Quantum deformations of generalised Kac-Moody algebras and their modules, J. of Algebra 175(1995), $1041-1066$.

11 K. Jeong, S.-J.Kang, M. Kashiwara, Crystal bases for quantum generalised Kac-Moody algebras and their modules, Proc. London Math.Soc.(3)90(2205),395-438.

${ }^{12}$ Li,F. and Duplij,S.,Weak Hopf algebras and singular solutions of quantum Yang-Baxter equation, Commun. Math. Phys.225(2002),191-217.

13 Nichols,W.D. and Taft,E.J. The left antipodes od a left Hopf algebra, Contemp.Math. 13(Ametrica Mathematical Society,Providence, RI,1982).

${ }_{14}$ Wu Zhixiang, The Weak Hopf Algebras Related to Generalized Kac-Moody Algebra, J.Math.Phs.(to appear).

15 Yang, S. ,Weak Hopf algebras corresponding to Cartan matrices, J.Math.Phys.46(2005),1-18.

16 Yamanouchi,T.,Duality for generalized Kacalgebras and a characterization of finite groupoid algebras, J.algebra,163(1994),950. 